\title{
HYPOTHESIS
}

\section{Influence of hypoxia in inflammatory synovitis}

\author{
T Bodamyali, C R Stevens, M E J Billingham, S Ohta, D R Blake
}

\begin{abstract}
Hypothesis
Persistent synovitis results from a hypoxia driven progressive transition to glycolytic metabolism that promotes transcriptional changes permissive to unresolved inflammation as the disease progresses. Erosion of cartilage and bone may involve gene expression characteristic to the "anoxic response system" of wound repair.
\end{abstract}

\section{HYPOXIA AND MODULATION OF IMMUNE}

RESPONSES

The pathogenesis of rheumatoid arthritis (RA) is hypothesised to involve inappropriate triggering of the MHC controlled immune surveillance, resulting in altered $\mathrm{T}$ and $\mathrm{B}$ cell profiles. Recently, the cytokine profile of T helper lymphocytes has been associated with the disease. The cytokine repertoire of inflamed synovia is categorised as that of a Th1 response. The characteristic Th1 response displays increased expression of select cytokines such as TGF $\beta$, IFN $\gamma, \mathrm{TNF} \alpha, \mathrm{IL} 1$, and IL2. In contrast, Th2 responses exhibit increased IL4, IL5, IL10, and IL13 (predominantly anti-inflammatory cytokines). While the levels of TNF $\alpha$ and IL1 are high within RA synovium, ${ }^{1}$ the level of IL2 is paradoxically low. ${ }^{2}$ This is atypical of a Th1 response, but may be accounted for by the overriding hypoxic condition of the RA synovium.

Hypoxia transcriptionally upregulates TNF and IL1 but downregulates IL2. ${ }^{34}$ The predominance of a given cytokine in the microenvironment of the responding Th cell influences Th1/Th2 differentiation. ${ }^{5}$ This phenomenon is believed to be regulated by phosphorylation of the relevant signal transducers and activators of transcription (STATs), after cytokine binding to their associated receptors. Based on the knowledge that hypoxia can modulate both cytokine expression and phosphorylation events, we propose that within inflamed synovial tissues, hypoxia underlies the functional polarisation of the Th1/Th2 lymphocytes and the apparent Th2 to Th1 switch in RA. The $\mathrm{T}$ cell cytokine profile of inflamed synovia may account for the development of an "unorganised" or abnormal inflammatory response. However, it does not convincingly explain the persistence of the existing inflammatory reaction, or more distant events such as the development of pannus tissue and the erosion of cartilage and bone. Evidence of an ancillary mechanism to cytokine induced inflammatory changes comes from a study of RA patients with HIV. In this situation, even when terminal loss of $T$ cells occurs and all immune and cytokine activity ceases (patients in remission) destructive rheumatoid pathology still continues. ${ }^{6}$

The apparent RA synovial $\mathrm{T}$ cell hyporesponsiveness (or anergy) may also be related to hypoxia. It is known that transient carbonylamino condensation, or Schiff base formation, regulates $\mathrm{T}$ cell and antigen presenting cell (APC) interactions. ${ }^{7}$ Inhibition of $\mathrm{T}$ cell responses can thus occur, depending on the species and concentration of Schiff base forming aldehydes. Such low molecular weight species are generated during hypoxic metabolism. We have demonstrated the presence of several low molecular mass carbonyl compounds, notably acetone and acetoacetate, in RA synovial fluid $(\mathrm{SF})^{8}$ that can potentially lead to the observed synovial $\mathrm{T}$ cell anergy.

Other studies indicate that the cytokine and growth factor profile of the Type A (macrophage) and Type B (fibroblastic) synoviocytes in culture, match more closely with that displayed by rheumatoid synovial tissues. ${ }^{9}$ These observations reflect a dominant and persistent cytokine and growth factor influence by resident synovial cells rather than the $T$ lymphocytes; in particular, macrophage or Type A derived IL1, IL6, TNF $\alpha$ and GM-CSF. The production of macrophage (Type A synoviocyte) derived cytokines is also influenced by hypoxia. ${ }^{3}$

We therefore propose that chronic hypoxia and anoxia are the most significant factors mediating persistent synovitis and bone erosions and should be considered as a direct target for therapeutic manipulation.

\section{RHEUMATOID SYNOVIUM IS CHRONICALLY}

HYPOXIC

The hypoxic nature of rheumatoid synovium was originally suggested on the basis of measurements of oxygen tension within the inflamed cavity. ${ }^{10}$ Other studies reporting raised carbon dioxide tension, lactate, lowered glucose and acidosis also supported these observations. ${ }^{11}$ More indirect, but supportive evidence was also provided by studies of the synovial oxidative metabolism, revealing increased metabolic demand and shift to glycolytic metabolism. ${ }^{12}$ These observations were confirmed with our studies of the synovial membrane and fluid from rheumatoid joints,
Accepted for publication 28 September 1998 
using nuclear magnetic resonance (NMR) spectroscopy. ${ }^{13}$ The results of this sensitive technique displayed a profile of low molecular weight metabolites consistent with hypoxic metabolism. Decreased synovial $\mathrm{pH}$, raised lactate, 3-D-hydroxybutarate, acetate levels, and ketone body formation confirmed a progressive shift to glycolytic metabolism, in accordance with chronic hypoxia. Further evidence came from studies utilising a polarographic needle electrode, which directly measured $\mathrm{PO}_{2}$ levels in diseased synovium and verified the hypoxic nature of inflamed synovia. ${ }^{14}$ The most hypoxic regions were found to be the innermost synovium in contact with the synovial fluid, irrespective of effusions. Our morphometric analysis of inflamed synovial tissues also supported these observations and revealed structural features that explain chronic hypoxia. ${ }^{15}$

Although synovitis was long considered to be an angiogenesis driven pathology, in our analyses, the capillary density was calculated to be a third of that in normal synovium. In addition, the average distance of the capillaries from the joint cavity was found to increase in rheumatoid synovia. These measurements indicate inadequate perfusion because of failure of angiogenesis to vascularise innermost synovium. We interpret this as the failure of angiogenesis to keep pace with synovial thickening and have discussed the implications in the context of inflammatory synovitis. ${ }^{16}$ Indeed, the prevalence of such events in inflamed rheumatoid synovium is exemplified by the presence of an avascular and predominantly hypoxic "pannus" tissue. We further argue that the loss of highly organised vascular structure causes relatively less uniform perfusion through the tissue. Subsequent poor blood flow aggravates hypoxia by diminishing the oxygen gradient out of the vessels and reduces the capillary release rate in small, remote capillaries. With sustained hypoxia, the capillaries become "paralysed" and lose their normal vasoactive responses. In addition to this hypoperfusion, mobility of the inflamed joint causes increased intra-articular pressure, further restricting synovial blood flow. ${ }^{17}$ Other hypoxia induced factors prevail. The presence of saturable, high affinity vascular endothelin (ET-1) binding sites within RA synovial sections and the localisation of ET-1like immunoreactivity to synovial microvascular endothelial cells, we believe, is a further indication of a hypoxia mediated contribution to the reduction in local synovial perfusion. ${ }^{18}$

Vascular insufficiency, hypoxia, and inflammatory cell infiltration leads to synovial fibroblastic hyperplasia with a resultant net increase in metabolic demand. The high metabolic demand predicts a predominantly glycolytic metabolism, a situation seen during wound repair in an essentially anoxic environment. ${ }^{19}$ Increased activities of enzymes such as glyceraldehyde-3-phosphate dehydrogenase, glucose-6-phosphate dehydrogenase, lactate dehydrogenase, and mitochondrial cytochrome oxidase within inflamed synovia demonstrate increased glycolytic activity. ${ }^{20}$ Increased intracellular but decreased extracellular sulphydryl content within the inflamed synovium is a strong indication of the disrupted redox balance. $^{21}{ }^{22}$ We suggest, this apparent redox imbalance in rheumatoid synovium is a manifestation of chronic hypoxia and movement induced, intermittent hypoxia/reperfusion.

\section{CELLULAR RESPONSES TO HYPOXIA}

A number of biochemical changes precede cell injury induced by hypoxia (reviewed by Khan and $\mathrm{O}^{\prime} \mathrm{Brien}^{23}$ ). These changes are consequences of the inactivation of oxidative phosphorylation in the electron transport chain. A significant drop in the ATP levels follow increases in cytosolic calcium and sodium, phospholipase $\mathrm{A}_{2}$ activation, and membrane phospholipid degradation. Increased lactate/ pyruvate ratios indicate a concomitant increase in the free cytoplasmic $\mathrm{NADH} / \mathrm{NAD}^{+}$ratios. Increased blood ketone body formation (acetoacetic/ $\beta$-hydroxybutyric acid ratio) is also an indicator of mitochondrial NADH/ $\mathrm{NAD}^{+}$ratio and redox potential. Increased $\mathrm{NADH}$ levels thus infer a reducing environment in which cells are hypoxia sensitive. This sensitivity often represents a switch to genetic programmes that control appropriate responses to prolonged changes in intracellular oxygen tension. At the cellular level, the initial impact of hypoxia is on the regulation of intermediary metabolism, affecting genes encoding enzymes responsible for glucose transport, glycolysis, and gluconeogenesis, regulating the biochemical responses discussed above.

\section{CELLULAR RESPONSES TO ANOXIA}

Anderson and Stoler have coined the term "anoxic response system" to describe events that occur at extremely low oxygen tensions observed in early wound sites. ${ }^{19}$ This system has been devised during evolution for the purpose of wound debridement and entails a switch to glycolytic metabolism, release of cathepsins and endonucleases to remove damaged and dead tissue. ${ }^{24}$

TISSUE RESPONSES TO HYPOXIA

At the tissue level, hypoxia induces coordinated activation of a network of genes that regulate processes leading to tissue reorganisation and regeneration. The resident cell types characterising the tissue, respond to hypoxia by a tightly controlled sequential process. The sequence of events begins with the "sensing" of the environmental change at the cellular level via signalling mechanisms involving the extracellular matrix and cell surface "sensor" molecules. The sensor then transduces the signal to the intracellular environment through second messenger systems that subsequently activate transcription factors (such as hypoxia inducible factor-1 (Hif-1)) and initiate specific transcriptional events. The nature and mechanisms of activation of this apparently universal "hypoxia sensor" has been reviewed by Bunn and Poyton. ${ }^{25}$ It has also long been established that the sensor is a haemoflavoprotein. ${ }^{26}$ 
HYPOXIA AND XANTHINE OXIDOREDUCTASE

Xanthine oxidoreductase, a three redox centred flavoprotein, generally believed to play a part in purine metabolism, is transcriptionally induced by hypoxia. ${ }^{27}$ The functional significance of this enzyme, specifically in synovitis, is still far from clear. However, several theories based on hypoxia/reperfusion injury, have attributed a pivotal role for this enzyme in pathology because of its capacity to generate reactive oxygen species. Recent observations relating to the signalling role of such ROS have indicated a "signal transducer" role for the enzyme, particularly in hypoxia/reperfusion related pathology.

The relevance of this enzyme in hypoxia driven pathology has been the subject of much debate since xanthine was considered to be the essential substrate for the enzyme mediated generation of ROS via reduction of molecular oxygen. It is now clear that the enzyme is also capable of using $\mathrm{NADH}$ as a substrate that facilitates the reduction of molecular oxygen to ROS. $^{28}{ }^{29}$ We discussed the evidence for the accumulation of $\mathrm{NADH}$ in hypoxic tissues. Under physiological conditions, the enzyme is believed to metabolise xanthine/hypoxanthine to urate with the aid of $\mathrm{NAD}^{+}$as the electron acceptor. It follows therefore, that this redox active enzyme (having three intact redox centres) is capable of maintaining the redox balance via alternately using $\mathrm{NAD}^{+}$as an electron acceptor and generating NADH as well as using $\mathrm{NADH}$ as a substrate during hypoxia, leading to ROS generation. The former mechanism feeds into the "salvage pathway" of purine metabolism, maintaining the nucleotide pool whereas the latter mechanism may act as a "signal transducer" for redox changes resulting from hypoxia. Such a versatile mechanism has previously been described for a bacterial Put A protein with a dual role in enzymatic metabolism as well as transcriptional control. ${ }^{30}$ Furthermore, we have recently reported that under conditions of hypoxia, this enzyme is also capable of generating nitric oxide from nitrate/nitrite ${ }^{31}$ which presents the enzyme as a suitable candidate for being a "hypoxia sensor" with redox mediated signalling capacity.

How can xanthine oxidoreductase act as a "redox sensor" and modulate transcriptional events downstream? Firstly, the predominant endothelial localisation of the enzyme indicates a strategic locality for it to act as a sensor of any $\mathrm{PO}_{2}$ changes as well as related metabolic alterations, such as $\mathrm{NADH} / \mathrm{NAD}^{+}$redox balance. Secondly, the enzyme has specific high affinity for sulphated glycosaminoglycans (GAGs), ${ }^{32}$ integral structural components of extracellular matrix. The sulphation pattern of GAGs is particularly influenced by hypoxia. ${ }^{33}$ In this context, xanthine oxidoreductase is a putative sensor for environmental changes transduced via the extracellular matrix GAGs. The presence of high circulating levels of this enzyme in various conditions, including rheumatoid arthritis, ${ }^{34}$ may also indicate the GAG mediated "trapping" or "recruitment" of this putative sensor where it is required to trans- duce the signal from an extracellular to intracellular environment via ROS production.

How can hypoxia/redox activated $\mathrm{XO}$ and $\mathrm{XO}$ derived ROS modulate subsequent intracellular events? The activation of a number of specifically hypoxia controlled transcription factors, such as Hif- $1,{ }^{35} \mathrm{AP}-1$, and NF- $\mathrm{BB}$, are all modulated by ROS driven redox events. ${ }^{3637}$ The localisation and functional capacity of xanthine oxidase to generate signalling molecules such as hydrogen peroxide and nitric oxide that can modulate hypoxia related transcription factors, has provided the basis for our current investigations on xanthine oxidase as an "oxygen sensor".

The net change in $\mathrm{PO}_{2}$ is signalled and transduced via the pathway described above, which leads to the production of associated proteins required for the achievement of homeostasis. At this level, the cell types that are most sensitive to $\mathrm{PO}_{2}$ changes go through necrosis or apoptosis. Altered cellular proteins, which are potentially toxic or antigenic, can be cleared in two ways. One pathway is via the induction of "heat shock" or "stress proteins", which either correct the protein alterations or act as chaperones to clear/recycle these potential toxins. Many of these stress proteins have hypoxia response elements (HRE) in their promoters, facilitating Hif-1 mediated activation. ${ }^{38}$

The second wave of reparative responses to hypoxia mediated localised cell death and related release of altered proteins, is the inflammatory process. The initial step in the recruitment of immunomodulatory cells is the induction of a whole host of adhesion molecules (integrins), which mediate the capture, rolling, adhesion, and transmigration of these cells from the circulation. The transcriptional induction of the genes for these integrin molecules, for the most part, involve hypoxia or redox controlled transcription factors. ${ }^{39}$

The recruited immunomodulatory cells, as well as dealing with the clearance of the antigenic proteins, release a range of growth factors and cytokines that lead to proliferative responses in fibroblasts. Macrophages also contribute to this process by releasing potent cytokines such as TNF $\alpha$ and TGF $\beta .^{340}$ Both hypoxia and TGF $\beta$ are known to induce ET-1 production from endothelial cells, although differential expression is observed in different vascular beds. ${ }^{41}{ }^{42}$ Fibroblastic cells are then primed by ET- 1 to produce factors that initiate extracellular matrix production and angiogenic factors, collagens, FGF, PDGF, and VEGF, thus aiding in the reorganisation and repair of the damaged tissue. ${ }^{43-45}$ The genes for these factors are controlled by hypoxia/redox sensitive transcription factors such as Hif-1, AP-1, and NfкB. ${ }^{24}$

At the whole body level, hypoxia influences the regulation of ventilation and red cell mass via transcriptional modulation of erythropoietin and tyrosine hydroxylase genes, which are also controlled by the transcription factor Hif- $1 .^{25}$ This represents a hypoxia driven global response for re-establishing normal perfusion. 
Table 1 Proteins and implicated transcription factors induced by hypoxia and anoxia

\begin{tabular}{|c|c|c|}
\hline Protein & Transcription factor & Reference \\
\hline \multicolumn{3}{|l|}{ Hypoxia induced } \\
\hline Xanthine oxidoreductase (XOR) & $\begin{array}{l}\text { Hypoxia inducible factor (Hif-1)-like element in } \\
\text { promoter region, NF-IL6 }\end{array}$ & 27 \\
\hline Haem oxygenase-1 (HO-1) & Hif-1 & 38 \\
\hline Glyceraldehyde-3-phosphate dehydrogenase & Hif-1 & 25 \\
\hline Lactate dehydrogenase & Hif-1 & 25 \\
\hline Erythropoietin (EPO) & Hif-1 & 25 \\
\hline Vascular endothelial growth factor (VEGF) & Hif-1, AP-1 & 25 \\
\hline Transforming growth factor $\beta$ (TGF $\beta)$ & Hif-1, Sp-1 & 50 \\
\hline Endothelin-1 (ET-1) & Activating protein-1(AP-1) & 51 \\
\hline Human $\alpha_{2}(\mathrm{I})$ collagen & $\mathrm{AP}-1, \mathrm{Sp}-1$ complex & 52 \\
\hline TNF $\alpha$, IL1, IL8, IL6 & NFKB & \\
\hline \multicolumn{3}{|l|}{ Anoxia induced } \\
\hline Lactate dehydrogenase $_{\mathrm{k}}$ & ? AIF & 48 \\
\hline Cathepsin D & ? AIF & 19 \\
\hline Cathepsin $\mathrm{L}$ & ? AIF & 19 \\
\hline Endonuclease NX & ? AIF & 26 \\
\hline
\end{tabular}

TISSUE RESPONSES TO ANOXIA

Anoxia results in the activation of the "anoxic response system" that plays an important part in clearing debris from a wound, before release of angiogenic factors and restoration of blood supply. As the oxygen tension rises, proteolytic enzyme release ceases and anoxic response gives way to matrix resynthesis leading to restitution of tissue lost during the wounding or trauma. ${ }^{25}$ In parallel with hypoxia mediated induction of Hif-1, anoxia induces a separate transcription regulatory element anoxia inducible factor (Aif), ${ }^{46}$ with a distinct set of target genes separate from those regulated by Hif- 1 . In rodents, the anoxic response system is characterised by the upregulation of retroviral inserts, VL30 retrotransposon elements, with unknown functions. ${ }^{46}$ These are only seen during wound repair and invasion of certain tumours in rodents, leading Anderson and Stoler to suggest that some tumours have "borrowed" the wound anoxic response system to invade normal tissue. It is conceivable that erosion of cartilage and bone in RA may involve this same anoxic response system. Interestingly, the MRL/lpr strain of mice that develop spontaneous erosive arthritis have a retrotransposon inserted in the fas gene, thereby interfering with apoptosis. ${ }^{47}$

We suggest that the slow, intermittent progression of cartilage and bone erosion is consistent with intermittent on/off cycles of the anoxic response system in a wound environment, but more studies are required to prove this.

In contrast, hypoxia induced events occurring on the other side of the synovial pannus, we believe, are responsible for the synovitis driven by induced inflammatory cytokines. See table 1 for a summary of hypoxia and anoxia induced proteins.

TRANSCRIPTIONAL AND PHENOTYPIC CHANGES IN RHEUMATOID SYNOVIUM REFLECT GENETIC RESPONSES TO A HYPOXIC/ANOXIC METABOLISM We have reviewed above the collective evidence supporting the chronically hypoxic nature of the inflamed rheumatoid synovium. Upregulation of the glycolytic enzymes glyceraldehyde3-phosphate dehydrogenase and lactate dehydrogenase in synovial tissues clearly reflect a hypoxia mediated metabolic change because the transcriptional control of such enzymes rely predominantly on the activation of Hif-1. In fact, Hif-1 is probably the most directly involved transcription factor in the activation of hypoxia responsive genes that are believed to play significant parts in the pathology of synovitis. The prime example of genes controlled by hypoxia through Hif-1 activation is the gene for VEGF. ${ }^{25}$ VEGF is a cytokine with strong influence on the regulation of wound healing, response to hypoxic injury, and tumour pathogenesis (the common element being the induction of angiogenesis). Recent evidence suggests a role for this cytokine in bone remodelling. ${ }^{48}$ Significant transcriptional upregulation of VEGF expression in rheumatoid synovial fibroblastic lining cells has also been reported. ${ }^{49}$ These observations, we suggest, again reflect a hypoxia driven attempt to establish the much required vascularisation to adequately perfuse the rheumatoid synovium.

Erythropoietin (EPO) production is another significant hypoxia regulated event that is controlled by the activation of Hif- $1 .{ }^{25}$ Studies analysing EPO production in RA have shown increased concentrations, again implying a possible hypoxia mediated impairment of the normal relation between EPO concentrations and the degree of anaemia.

Several other genes that are transcriptionally modulated by hypoxia have significant contribution to the pathology of synovitis. These include the genes for cytokines such as TGF $\beta$, which mediate both inflammatory and bone resorptive events, and the vasoactive, fibroblastic mitogen ET-1. Hypoxia induces TGF $\beta$ through pathways common to hypoxia sensing mechanism(s) described for EPO. ${ }^{50} \mathrm{TGF} \beta$, in turn, is known to regulate ET-1 expression via Fos and Jun oncoproteins (the transcription factor AP-1). ${ }^{51}$ TGF $\beta$ also mediates expression of collagen genes, which contain the AP-1 binding element. ${ }^{52} \mathrm{AP}-1$ is regulated directly by hypoxia/redox-balance. ${ }^{53} \mathrm{NF \kappa B}$, also a redox controlled transcription factor, regulates the transcription of the integrins. Inflamed synovial endothelial cells show increased surface expression of a series of integrin molecules (ELAM-1/ICAM-1). ${ }^{54}$ Lymphocytes and neutrophils within inflamed synovial tissues also show increased expression of the associated 
integrins (LFA-1, VCAM-1, VLA-4, and CD11a/CD18 respectively)..$^{55}$

Collectively, the reports on increased expression of these components within RA synovium indicate synovial hypoxia as being a pivotal factor in fibroproliferative and matrix synthesis inducing events, leading to pannus formation.

Some of the most important protein factors that control hypoxia/redox mediated events are proto-oncogenes. The potent fibroblastic growth factor, PDGF is identical to the protooncogene c-sis, which confers altered growth characteristics to target cells. ${ }^{56}$ Increased expression of c-sis has been reported in RA synoviocytes. ${ }^{57}$ Within the chronically hypoxic innermost layer (pannus tissue) and erosive sites, fibroblastic lining cells have a transformed morphology, show anchorage independent growth characteristics and increased proliferation. ${ }^{58}$ This, we correlate with the increased secretion of PDGF from the fibroblastic cells, as an autocrine factor within the inflamed synovium. Similarly, the transcription factor AP-1, is the protein product of c-fos and c-jun proto-oncogenes. Increased expression of these proto-oncogenes in inflamed synovial sections has also been reported. ${ }^{59}$

Other proto-oncogene related transcription factors, such as NFkB, are modulated by hypoxia mediated downstream events associated with redox balance and oxidant stress. A functionally significant component of $\mathrm{NFkB}$, is the proto-oncogene c-rel. Increased activation of this hypoxia/redox controlled transcription factor and its predominant vascular localisation within the inflamed synovium further emphasises the pivotal influence of hypoxia in the disease. ${ }^{60}$ We suggest therefore, that hypoxia/ redox controlled oncogenic activity and the control of associated transcription factors and their target genes, largely explain the structural and phenotypic changes within the inflamed synovium.

In parallel to hypoxia controlled genes within the inflamed synovium, anoxia plays a significant part in transcriptional events at bone and cartilage erosive sites-that is, the pannus tissue. Descriptions by Gay and his colleagues of a so called "transformed fibroblast phenotype" within pannus tissue with the property of eroding cartilage and bone in RA, ${ }^{61}$ are very similar to those of the activation state of the wound fibroblast of the "anoxic response system". At the eroding interface of the pannus, transformed fibroblasts have been shown to release cathepsins B, L and, importantly, $\mathrm{K}$, which is the isotype released by osteoclasts during bone resorption. ${ }^{62}$ These cells express the adhesion molecule VCAM-1, which is thought to play a part in adhesion of the transformed fibroblast to the tissue being resorbed. Similar studies to compare wound fibroblasts remain to be undertaken because the process of erosion has overtones of the process of wound repair by the fibroblast in anoxia and may be a quite separate process to the hypoxia driven cytokine and immune events on the cavity side of the synovial membrane. Telling evidence for this comment derives from observation on patients with
AIDS who also have the misfortune to suffer from RA. At the time CD4+ lymphocytes fall precipitously, symptoms of the arthritis disappear, however, at necropsy evidence was found that erosion was still continuing in the absence of cytokine stimulation of the eroding fibroblasts. ${ }^{6}$

Ingenious transfection studies of RA transformed fibroblasts with the IL1 inhibitor IRAP (IL1 receptor antagonist protein), and coimplantation of these with human cartilage in a SCID mouse system, demonstrated that the erosive process through cartilage is not driven by the cytokine IL1. The transfected cells eroded cartilage at the same rate as those with an irrelevant construct, however, the accompanying chondrocytic chondrolysis was inhibited. ${ }^{63}$ These cells are able to produce low levels of cytokines, but do not seem to respond to such cytokines. Finally, the first cells within the joint to proliferate proliferate in the MRL/ lpr mouse model of erosive arthritis are the synovial fibroblasts, and the erosive process is underway before any inflammatory and immune cell involvement. ${ }^{64}$

The "physiological" cell and tissue responses to hypoxia/anoxia are adaptive and organised, and lead to the resolution of injury and homeostasis. Although the pathological characteristics of synovitis parallel these physiological responses to hypoxia/anoxia, lack of resolution of injury implies a specific disruption of such organised and tightly controlled responses in synovitis. We argue that movement of inflamed joints introduce mechanical factors as well as pressure changes that contribute to chronic hypoxia and localised anoxia (particularly at erosive sites), preventing the completion of much required angiogenesis. Thus, the net effect of hypoxia and/or anoxia induced changes within the rheumatoid joints is persistent, unresolved inflammation and cartilage/ bone destruction.

\section{THERAPEUTIC APPROACHES}

An extensive array of therapeutic strategies have been suggested and tested for the treatment of RA, albeit with no definitive outcome. Some approaches have been based on the antioxidant/oxidant imbalance in inflamed synovial tissues. Supplementation with folic acid and the antioxidant vitamin $\mathrm{E}$, have been one such approach. Previous studies have not conclusively demonstrated that the rheumatoid joint is locally folate deficient, or that, if such is the case, folate gets into the joint when given as an oral supplement. Folate given intraarticularly however, was found to suppress some of the hyaluronan induced flares. Further pharmacokinetic studies are required for the elucidation of the role of folate in rheumatoid synovitis.

In contrast, it is known that patients with RA are locally deficient of vitamin $\mathrm{E}$ and that the latter gets into the joint when given orally. ${ }^{65}$ However, a significant anti-inflammatory effect has not yet been reported. Antioxidants and vitamins act in concert with each other and supplementing one without the other may actually deplete tissues of certain vitamins. It is 
also possible that other antioxidants are depleted in patients with RA. Indeed, it is known that patients with RA are vitamin $C$ deficient. ${ }^{66}$ Clearly, if vitamin $\mathrm{C}$ regenerates vitamin $\mathrm{E}$ in vivo as it does in vitro, patients who are deficient in both vitamins have little chance of benefiting if one is given without the other. Vitamin E does however have an analgesic effect that seems to be independent of its antioxidant properties. The interactions between the various antioxidant enzymes in vivo have yet to be fully elucidated, and there remains much scope for clinical trials using combinations of these compounds.

Alternative considerations have included "mopping up" the toxic products of oxidative injury, such as formate. Although raised levels of formate have been found in the rheumatoid joint, the precise part this molecule plays in rheumatoid synovitis is far from clear. ${ }^{67}$ However, the fact that a flare can be induced by the intra-articular injection of hyaluronan in adjuvant arthritis in the rabbit, suggests that formate may at least be contributing to acute synovitis. $^{68}$

Relatively recent strategies involve anticytokine treatment. Clinical trials using chimeric anti-TNF $\alpha$ monoclonal antibodies, in open label and randomised placebo controlled studies, have demonstrated a dose dependent efficacy with significant improvement in disease activity. ${ }^{69}$ However, the possible long term effects of such treatment are largely unknown.

The alternative to counteracting oxidative stress or inhibiting specific cytokines, we believe, is to use the established and substantiated observations that the rheumatoid synovium is hypoxic. On this basis, we suggest development of novel therapeutic strategies that target hypoxia directly.

The direct killing of hypoxic cells has long been the concern of radiotherapists and oncologists, as hypoxic cells are radiation resistant compared with oxic cells, and this resistance is an important factor influencing local tumour control by radiation. The hypoxic intracellular environment is a reducing one, as we eluded to earlier. This reductive metabolism can be used by certain drugs that are reduced to more toxic metabolites than the parent drug. Such "bioreductive" drugs have considerably more toxic effects on hypoxic than oxic mammalian cells, and their cytotoxicity correlates with their electron affinity. The basis for their "selective" toxicity towards hypoxic tissue is ascribed to the activation of the drug to a free radical intermediate. In fully oxygenated tissues, the free electron from the reduced drug is rapidly received by cellular oxygen via the process of "futile cycling". The leakage of superoxide anion $\left(\mathrm{O}_{2}{ }^{\circ}\right)$ is of potential concern but is suppressible by the antioxidant mechanisms of normal cells.

The critical step in hypoxic cytotoxicity is enzyme mediated reductive activation. A decrease in cellular $\mathrm{PO}_{2}$ is known to induce the synthesis of reductase enzymes such as xanthine oxidoreductase, NADPH:cytochrome $\mathrm{P}_{450}$ reductase, and i-NOS. Indeed, hypoxia has been identified to be a significant factor in the transcriptional regulation of these particular enzymes. In addition, other factors prevalent within the hypoxic environment of the inflamed rheumatoid joint, such as calcium mobilisation and cytokine release, are also known to activate these enzymes. We have previously reviewed the significance of these enzymes in RA synovium. ${ }^{70}$

One particular bioreductive drug, metronidazole, has previously been used in RA with reports on positive therapeutic effects. ${ }^{71}$ This was based on the postulate that RA may be caused by anaerobic bacteria. However, these studies were not fully controlled. Furthermore, a double blind study in a mixed rheumatoid population did not confirm a therapeutic action. Metronidazole is, however, the least active of the nitro-heterocyclic compounds, and it is reasonable to speculate that the rheumatoid synovium is a potential target for other bioreductives with a greater electron affinity. A range of bioreductive drugs are yet to be tested in rheumatoid patients. Our current in vivo studies have positive primary indications. The possibility of targeted, intra-articular administration of such cytotoxic bioreductive drugs would result in "auto-synovectomy" and avoid the need for painful and lengthy surgery. An additional advantage of such a treatment regimen would be to minimise the use of potentially more toxic drugs that are currently the mainstay of RA treatment.

Our recent strategy is based on the potential use of bioreductives as "carrier" molecules to specifically target other drugs to the synovium. This would not only have the effect of reducing the risk of systemic side effects, but also increase the therapeutic effect of the "targeted drug" linked to the "carrier". For example, the weak acidic based NSAIDs, which undergo ion trapping in acidotic tissue could be linked to a bioreductive agent and targeted to hypoxic or anoxic cells, achieving improved efficacy and minimal toxicity. Corticosteroids may also be covalently linked to bioreductive compounds, again improving the therapeutic index and minimising systemic corticosteroid effects. We are currently designing the synthesis of such bioreductively linked novel therapeutic agents to be tested in our model systems.

Funding: The Bone and Joint Research Group at the University of Bath receives programme grant support from the Arthritis and Rheumatism Council for Research.

1 Tetta C, Camussi G, Modena V, Di Vittoro C, Baglioni C. Tumour necrosis factor in serum and synovial fluid of patients with active and severe rheumatoid arthritis. Ann patients with active and seve
Rheum Dis 1990;49:665-7.

2 Westacott CI, Whicher JT, Barnes IC, Thompson D, Swan AJ, Dieppe PA. Synovial fluid concentration of five different cytokines in rheumatic diseases. Ann Rheum Dis 1990;49:676-81.

3 Ghezzi P, Dinarello CA, Bianchi M, Rosandich ME, Repine $\mathrm{JE}$, White CW. Hypoxia increases production of nterleukin-1 and tumour necrosis factor by human mononuclear cells. Cytokine 1991;3:189-94.

4 Zukerberg AL, Goldberg LI, Lederman HM. Effects of hypoxia on interleukin-2 mRNA expression by $\mathrm{T}$ lymphocytes. Crit Care Med 1994;22:197-203.

5 Romagnani S. The Th1/Th2 paradigm. Immunol Today 1997;18:263-6.

6 Muller-Ladner U, Kriegsmann J, Gay RE, Koopman WJ, Gay S, Chatham WW. Progressive joint destruction in a human immunodeficiency virus-infected patient with human immunodeficiency virus-infected patient with

7 Gao XM, Rhodes J. An essential role for constitutive base-forming ligands in antigen presentation to murine T-cell clones. J Immunol 1990;144:2883-90. 
8 Naughton DP, Haywood R, Blake DR, Edmonds S, Hawkes GE, Grootveld M. A comparative evaluation of the metabolic profiles of normal and inflammatory knee-joint synovial fluids by high resolution proton NMR spectroscopy. FEBS Lett 1993;332:221-5.

9 Wagner S, Fritz P, Einsele H, Sell S, Saal JG. Evaluation of synovial cytokine patterns in rheumatoid arthritis and osteoarthritis by quantitative reverse transcription polymerase chain reaction. Rheumatol Int 1997;16:191-6.

10 Lund-Olsen K. Oxygen tension in synovial fluids. Arthritis Rheum 1970;13:769-76.

11 Treuhaft PS, McCarty DJ. Synovial fluid $\mathrm{pH}$, lactate, oxygen and carbon dioxide partial pressures in various join diseases. Arthritis Rheum 1971;14:475-84.

12 Dingle JTM, Page-Thomas DP. In vitro studies on human synovial membrane. A metabolic comparison of normal and rheumatoid tissue. Br J Exp Pathol 1956;37:318-36.

13 Naughton D, Whelan M, Smith EC, Williams R, Blake DR, Grootveld M. An investigation of the abnormal metabolic status of synovial fluid from patients with rheumatoid status of synovial fluid from patients with rheumatoid
arthritis by high field proton nuclear magnetic resonance arthritis by high field proton nuclear magn

14 Ellis GA, Edmonds SE, Gaffney K, Williams RB. Synovial tissue oxygenation profile in inflamed and non-inflamed knee joints. [Abstract].Br J Rheumatol 1994;33 (suppl 1): 172

15 Stevens CR, Blake DR, Merry P, Revell PA, Levick JR. A comparative study by morphometry of the microvasculature in normal and rheumatoid synovium. Arthritis Rheum 1991;34:1508-13.

16 Stevens CR, Williams R, Blake DR. Hypoxia and inflammatory synovitis: observations and speculations. Ann Rheum Dis 1991:50:124-32.

17 Merry P, Williams R, Cox N, King JB, Blake DR. Comparative study of intra-articular pressure dynamics in joints with tive study of intra-articular pressure dynamics in joints with acute traumatic and chronic inflammatory effusions: potential implications for hyp
Rheum Dis 1991;50:917-20.

18 Wharton J, Rutherford RAD, Walsh DA, Mapp PI, Knock GA, Blake DR, et al. Autoradiographic localisation and analysis of endothelin-1 binding sites in human synovia tissue. Arthritis Rheum 1992;35:894-9.

19 Anderson GR, Volpe CM, Russo CA, Stoler DL, Miloro $\mathrm{SM}$. The anoxic fibroblast response is an early-stage wound healing program. J Surg Res 1995;59:666-74.

20 Henderson B, Bitensky L, Chayen J. Glycolytic activity in human synovial lining cells in rheumatoid arthritis. Ann Rheum Dis 1979;38:63-7.

21 Butcher RG, Bitensky L, Cashman B, Chayen J. Differences in the redox balance in human rheumatoid and nonrheumatoid synovial lining cells. Beitr Pathol 1973;148: 265-274.

22 Hall ND, Blake DR, Bacon PA. Serum sulphydryl levels in early synovitis. J Rheumatol 1982;9:593-6.

23 Khan S, O'Brien PJ. Modulating hypoxia-induced hepatocyte injury by affecting intracellular redox state. Biochim Biophys Acta 1995;1269:153-61.

24 Anderson GR, Stoler DL. Anoxia, wound healing, VL30 elements, and the molecular basis of malignant conversion. Bioessays 1993;15:265-72.

25 Bunn HF, Poyton RO. Oxygen sensing and molecular adaptation to hypoxia. Physiol Rev 1996;76:839-85.

26 Goldberg MA, Dunning SP, Bunn F. Regulation of the erythropoietin gene: evidence that the oxygen sensor is a heme protein. Science 1988;242:1412-15

27 Terada LS, Piermattei D, Shibao GN, McManaman JL, Wright RM. Hypoxia regulates xanthine dehydrogenase Wright RM. Hypoxia regulates xanthine dehydrogenase activity at pre-and posttran

28 Sanders SA, Eisenthal R, Harrison R. NADH oxidase activity of human xanthine oxidoreductase-generation of superoxide anion. Eur J Biochem 1997;245:541-8

29 Zhang Z, Blake DR, Stevens CR, Kanczler JM, Winyard $\mathrm{PG}$, Symons MC, et al. A reappraisal of xanthine dehydrogenase and oxidase in hypoxic reperfusion injury:The role of NADH as an electron donor. Free Radic Res 1998 28:151-64

30 Ostrovsky de Spicer P, Maloy S. PutA protein, a membraneassociated flavin dehydrogenase, acts as a redox-dependent transcriptional regulator. Proc Natl Acad Sci USA 1993;90:4295-8.

31 Millar TM, Stevens CR, Benjamin N, Eisenthal R, Harrison $R$, Blake DR. Xanthine oxidoreductase catalyses the reduction of nitrates and nitrite to nitric oxide under hypoxic tion of nitrates and nitrite to nitric oxide
conditions. FEBS Letts 1998;427:225-8.

32 Radi R, Rubbo H, Bush K, Freeman BA. Xanthine oxidase binding to glycosaminoglycans:kinetics and superoxide dismutase interactions of immobilized xanthine oxidaseheparin complexes. Arch Biochem Biophys 1997;339:12535 .

33 Rivard CH. Effects of hypoxia on the embryogenesis of congenital vertebral malformations in the mouse. Clin Orthop 1986;208:126-30.

34 Jawed S, McCurdie I, Stevens CR, Grootveld M, Harrison $\mathrm{R}$, Blake DR. The role of elevated circulating plasma NADH oxidase activity in rheumatoid arthritis. Arthritis Rheum 1997;40:1323.

35 Wang GL, Semenza GL. General involvement of hypoxiainducible factor-1 in transcriptional response to hypoxia. Proc Natl Acad Sci USA 1993;90:4304-8.

36 Eric Huang L, Arany Z, Livingston DM, Bunn HF. Activation of hypoxia-inducible transcription factor depends primarily upon redox-sensitive stabilisation of its $\alpha$ subunit. J Biol Chem 1996;271:32253-9.
37 Schulze-Osthoff K, Bauer MK, Vogt M, Wesselborg S. Oxidative stress and signal transduction. Int J Vitam Nutr Res dative stress and

38 Lee PJ, Jiang B-H, Chin BY, Iyer NV, Alam J, Semenza GL, et al. Hypoxia inducible factor-1 mediates transcriptional activation of the heme oxygenase-1 gene in response to hypoxia J Biol Chem 1997;272:5375-81.

39 Cominacini L, Garbin U, Fratta Pasini A, Paulon T, Davoli A, Campagnola $\mathrm{M}$, et al. Lacidipine inhibits the activation of the transcription factor NF-kappaB and the expression of adhesion molecules induced by pro-oxidant signals on endothelial cells. J Hypertens 1997;15 pt 2):1633-40.

40 Schultz GS, Grant MB. Neovascular growth factors. Eye 1991;5:170-80

41 Kourembanas S, Marsden PA, McQuillan LP, Faller DV. Hypoxia induces endothelin gene expression and secretion in cultured human endothelium. J Clin Invest 1991;88: 1054-7

42 Brown MR, Vaughan J, Jimenez LL, Vale W, Baird A. Transforming growth factor-beta:role in mediating seruminduced endothelin production by vascular endothelial cells. Endocrinology 1991;129:2355-60.

43 Gutierrez S, Palacios I, Egido J, Gomez-Garre D, Hernandez P, Gonzalez E, et al. Endothelin-1 induces loss of proteoglycans and enhances fibronectin and collagen production in cultured rabbit synovial cells. Eur J Pharmacol 1996;302:191-7.

44 Nakamura T, Ebihara I, Fukui M, Tomino Y, Koide H. Effect of a specific endothelin receptor A antagonist on mRNA levels for extracellular matrixcomponents and growth factors in diabetic glomeruli. Diabetes 1995;44: $895-9$

45 Pedram A, Razandi M, Hu RM, Levin. Vasoactive peptides modulate vascular endothelial cell growth factor production and endothelial cell proliferation and invasion. J Biol Chem 1997;272:17097-103.

46 Estes SD, Stoler DL, Anderson GR. Anoxic induction of a sarcoma virus-related VL30 retrotransposon is mediated by a cis-acting element which binds hypoxia-inducible factor - and an anoxia-inducible factor. J Virol 1995;69: factor-and

47 Chu JL, Drappa J, Parnassa A, Elkon KB. The defect in Fas mRNA expression in MRL/lpr mice is associated with
insertion of the retrotransposon, Etn. J Exp Med insertion of the

48 Harada S, Rodan SB, Rodan GA. Expression and regulation of vascular endothelial growth factor in osteoblasts. Clin Orthop 1995;313:76-80

49 Jackson JR, Minton JA, Ho ML, Wie N, Winkler JD. Expression of vascular endothelial growth factor in synovial fibroblasts is induced by hypoxia and interleukin-1 beta. J Rheumatol 24:1253-9.

50 Gleadle JM, Ebert BL, Firth JD, Ratcliffe PJ. Regulation of angiogenic growth factor expression by hypoxia, transition
metals, and chelating agents. Am J Physiol 1995;268: C1362-8.

51 Lee ME, Dhadly MS, Tamizer DH, Clifford JA, Yoshizumi $M$, Quertermous T. Regulation of endothelin-1 gene expression by Fos and Jun. J Biol Chem 1991;266: 19034-9.

52 Chung KY, Agarwal A, Uitto J, Mauviel A. An AP-1 binding sequence is essential for regulation of the human alpha2(I) collagen (COL1A2) promoter activity by transforming growth factor-beta. J Biol Chem 1996;271:3272-8.

53 Meyer M, Schreck R, Baeuerle PA. H2O2 and antioxidants have opposite effects on activation of NFKB and AP-1 in intact cells:AP-1 as secondary antioxidant-responsive factor. EMBO J 1993;12:2005-15.

54 Abbot SE, Kaul A, Stevens CR, Blake DR. Isolation and culture of synovial microvascular endothelial cells: characterisation, and assessment of adhesion molecule expression. Arthritis Rheum 1992;35:401-6.

55 Haskard DO. Cell adhesion molecules in rheumatoid arthritis. Curr Opin Rheumatol 1995;7:229-34.

56 Doolittle RF, Hunkapiller MW, Hood LE, DeVare SG, Robbins KC, Aaronson SA, et al. Simian sarcoma virus onc gene, $\mathrm{v}$-sis, is derived from the gene (or genes) encoding a platelet-derived growth factor. Science 1983;221: $275-6$.

57 Remmers EF, Sano H, Lafyatis R, Case JP, Kumkumian $\mathrm{GK}$, Hla T, et al. Production of platelet derived growth factor B chain (PDGF-B/c-sis) mRNA and immunoreactive PDGF B-like polypeptide by rheumatoid synovium: PDGF B-like polypeptide by rheumatoid synovium: factor-1. J Rheumatol 1991;18:7-13.

58 Case JP, Lafyatis R, Remmers EF, Kumkumian GK, Wilder RL. Transin/stromelysin expression in rheumatoid synovium. A transformation-associated metalloproteinase secreted by phenotypically invasive synoviocytes. Am J Pathol 1989;135:1055-64.

59 Kinne RW, Boehm S, Iftner T, Aigner T, Vornehm S, Wewloh G, et al. Synovial fibroblast-like cells strongly express un-B and c-fos proto-oncogenes in rheumatoid- and osteoarthritis. Scand J Rheumatol 1995;101:121-5.

60 Marok R, Winyard PG, Coumbe A, Kus ML, Gaffney K, Blades S, et al. Activation of the transcription factor nuclear factor-kappa B in human inflamed synovial tissue. Arthritis Rheum 1996;39:583-91.

61 Muller-Ladner U, Kriegsmann J, Franklin BN, Matsumoto S, Geiler T, Gay RE, et al. Synovial fibroblasts of patients with rheumatoid arthritis attach to and invade normal human cartilage when engrafted into SCID mice. Am J Pathol 1996;149:1607-15. 
62 Sainsbury ISS, Hummel K, Bisbinas I, Lalor PA, Aberman HM, Learmonth ID, et al. Characteristics of fibroblasts at the interface between bone and pseudosynovium in aseptically loosened prostheses. Br J Rheumatol 1998, 37:S193.

63 Muller-Ladner U, Roberts CR, Franklin BN, Gay RE, Robbins PD, Evans $\mathrm{CH}$, et al. Human IL-1Ra Gene Transfer into Human Synovial Fibroblasts is Chondroprotective. J Immunol 1997;158:3492-8.

64 O'Sullivan FX, Fassbender HG, Gay S, Koopman WJ. Etiopathogenesis of the rheumatoid arthritis-like disease in MRL/1 mice. I. The histomorphologic basis of join destruction. Arthritis Rheum 1985;28:529-36.

65 Fairburn K, Grootveld M, Ward RJ, Abiuka C, Kus M, Williams RB, et al. Alpha-tocopherol, lipids and lipoproteins in knee-joint synovial fluid and serum from patients with inflammatory joint disease. Clin Sci (Colch) 1992;83:65764.

66 Lunec J, Blake DR. The determination of dehydroascorbic acid and ascorbic acid in the serum and synovial fluid of patients with rheumatoid arthritis (RA). Free Radic Res patients with rheumato
67 Grootveld M, Henderson EB, Farrell A, Blake DR, Parkes $\mathrm{HG}$, Haycock P. Oxidative damage to hyaluronate and glucose in synovial fluid during exercise of the inflamed rheumatoid joint. Detection of abnormal low-molecular-mass metabolites by proton-n.m.r. spectroscopy. Biochem J 1991;273:459-67.

68 Henderson EB, Grootveld M, Farrell A, Smith EC, Thompson PW, Blake DR. A pathological role for damaged hyaluronan in synovitis. Ann Rheum Dis 1991;50:196-200.

69 Maini RN, Elliott MJ, Brennan FM, Williams RO, Chu CQ, Paleolog E, et al. Monoclonal anti-TNF alpha antibody as a probe of pathogenesis and therapy of rheumatoid disease. Immunol Rev 1995; 144:195-223.

70 Kanczler JM, Sahinoglu T, Stevens CR, Blake DR. The complex influences of reactive oxygen species on rheumatoid erosions and synovitis. In: Hukkanen MVJ, Polak JM, Hughes SPF, eds. Nitric oxide in bone and joint disease. Cambridge: Cambridge University Press, 1998.

71 Marshall DA, Hunter JA, Capell HA. Double blind placebo-controlled study of metronidazole as a disease modifying agent in the treatment of rheumatoid arthritis. modifying agent in the treatment of

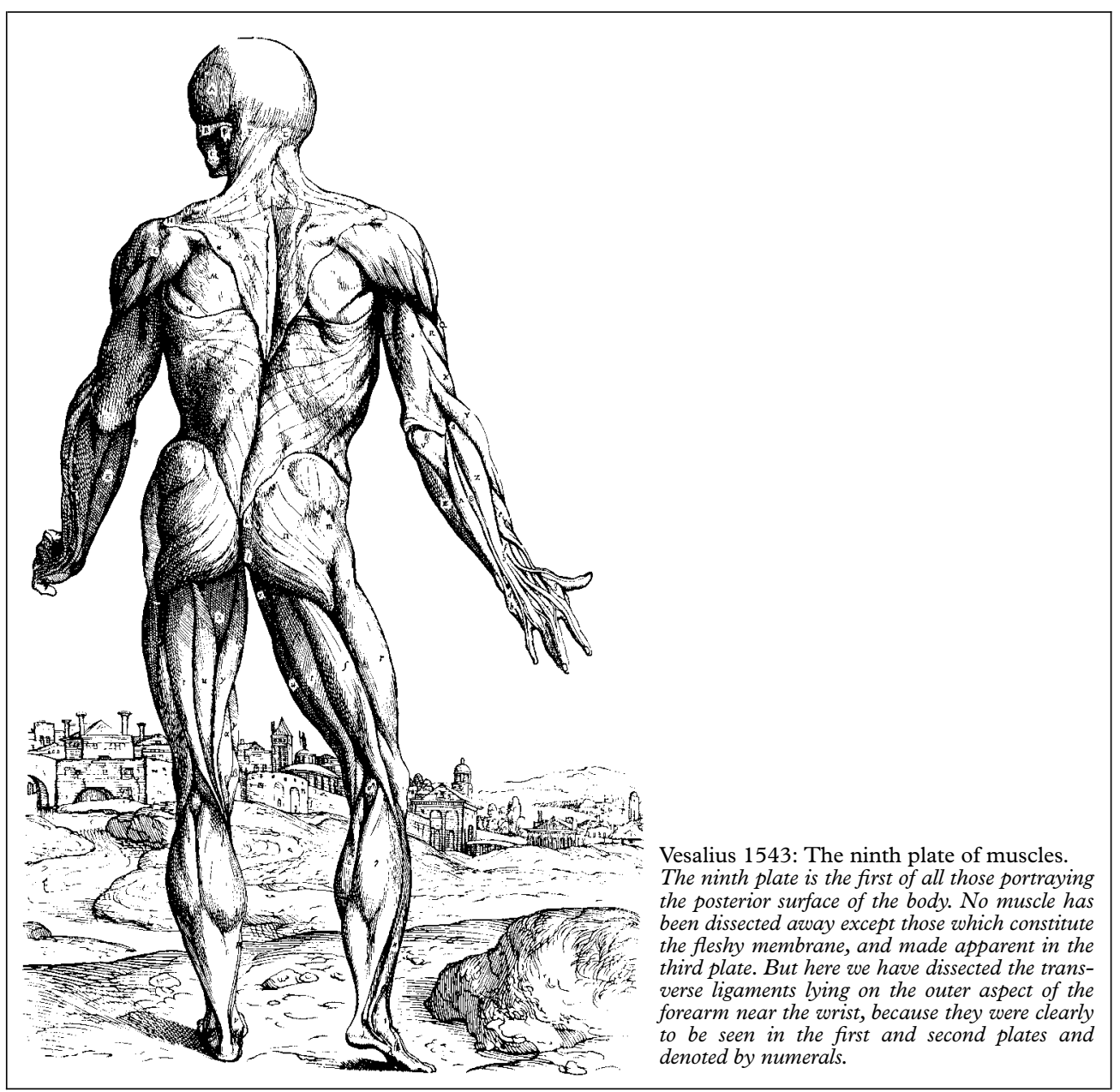

